\title{
Threshold of Inotropic Score and Vasoactive-Inotropic Score for Predicting Mortality in Pediatric Septic Shock
}

\author{
Dipu Kallekkattu ${ }^{1} \cdot$ Ramachandran Rameshkumar $^{1}\left[\right.$ D $\cdot$ Muthu Chidambaram ${ }^{1} \cdot$ Kandamaran Krishnamurthy $^{2}$. \\ Tamil Selvan ${ }^{1} \cdot$ Subramanian Mahadevan ${ }^{1}$
}

Received: 15 January 2021 / Accepted: 7 June 2021 / Published online: 27 July 2021

(c) Dr. K C Chaudhuri Foundation 2021

\begin{abstract}
Objective To determine the threshold of the inotropic score (IS) and vasoactive-inotropic score (VIS) for predicting mortality in pediatric septic shock.

Method This retrospective cohort study included children aged 1 mo to 13 y with septic shock, requiring vasoactive medication. The area under curve receiver operating characteristic (AUROC) was calculated using mean IS and mean VIS to predict PICU mortality, and Youden index cut points were generated. Sensitivity, specificity, and binary regression analysis were performed.

Results A total of 176 patients were enrolled (survivor, $n=72,41 \%$ and nonsurvivor, $n=104,59 \%$ ). For predicting the PICU mortality, AUROC (95\% CI) of IS was 0.80 (0.74-0.86) [sensitivity of 88.5 (80.7-94) and specificity of 58.3 (46.1-69.8)] and AUROC of VIS was $0.88(0.82-0.92)$ [sensitivity of 83.7 (75.1-90.2) and specificity of $80.6(69.5-89)$ ]. The respective cutoff scores of IS and VIS were 28 and 42.5. On regression analysis (adjusted odds ratio, 95\% CI), illness severity (PRISM-III) $(1.12,1.05-1.12)$, worst lactate value $(1.31,1.08-1.58)$, IS ( $>28)(3.98,1.24-12.80)$, and VIS (> 42.5) (4.66, $1.57-13.87)$ independently predicted the PICU mortality $\left(r^{2}=0.625\right)$.

Conclusion Threshold of inotropic score ( $>28)$ and vasoactive-inotropic score $(>42.5)$ were independently associated with PICU mortality. In addition to IS and VIS, severity and worst lactate value independently predicted septic shock mortality in PICU.
\end{abstract}

Keywords Children $\cdot$ Pediatric intensive care $\cdot$ Blood transfusion $\cdot$ Mortality $\cdot$ Morbidity $\cdot$ Septic shock $\cdot$ Vasoactiveinotropic score $\cdot$ Predictors

\section{Introduction}

Despite the improvement in sepsis care, the sepsis-related mortality rate was as high as $58 \%$, especially in the underfive age group in low-middle income countries (LIMC) [1, 2]. Fluid resuscitation is a fundamental intervention in pediatric septic shock management. The FEAST trial from LMIC demonstrated an increased $48 \mathrm{~h}$ mortality rate in children who received fluid boluses [3]. Recently, sepsis-induced

Ramachandran Rameshkumar

krramesh_iway@yahoo.co.in

1 Division of Pediatric Critical Care, Department of Pediatrics, Jawaharlal Institute of Postgraduate Medical Education and Research (JIPMER), Puducherry 605006, India

2 Pediatric Intensive Care Unit, University of West Indies, Wanstead, Barbados myocardial dysfunction is widely recognized in pediatric septic shock [4]. The degree of myocardial dysfunction and the amount of vasoactive (inotropic) therapy needed to achieve and maintain organ perfusion can predict clinical outcomes $[4,5]$. Frequent screening of myocardial dysfunction by echocardiography may not be possible in a resourcelimited setting. A bedside vasoactive/inotropic score that accurately quantifies the medication support points towards the degree of myocardial dysfunction [6]. Inotropic score (IS) was initially developed and used in pediatric postoperative cardiac patients [5]. IS was modified by adding commonly used other inotropic and vasoactive agents called vasoactive-inotropic score (VIS), giving equal weightage to each medication according to the potency [7]. The highest VIS score in the immediate postoperative period predicted a poor outcome [8]. Initially used in postoperative cardiac patients, VIS has been extrapolated to critically ill 
noncardiac patients. Few studies have validated the use of VIS in general pediatric intensive care unit (PICU) and pediatric septic shock [9-11]. Limited studies explored the VIS cutoff $(>20)$ and mortality in pediatric septic shock, especially from LMIC [12]. The present study aimed to define the IS and VIS cutoff to predict pediatric septic shock mortality in PICU.

\section{Material and Methods}

This retrospective cohort study was undertaken in the PICU of a tertiary care teaching hospital from July 2017 to June 2018. The institutional ethics committee approved the study with a waiver of written consent. Case records of children aged 1 mo to $13 \mathrm{y}$ with septic shock and requiring vasoactive therapy were enrolled. Children who received vasoactive agent therapy for $>6 \mathrm{~h}$ before admission and those with $\geq 2$ organ dysfunction at admission were excluded. Septic shock was managed as per unit protocol, which was adopted from the surviving sepsis campaign (SSC) and American college of critical care medicine (ACCM) guidelines [13, 14]. Sepsis and septic shock were defined using the international consensus conference on pediatric sepsis criteria [15]. The authors' unit is equipped with multimodal hemodynamic monitoring, including echocardiography (echo), and has the facility for kidney replacement therapy (KRT). There is no standby extracorporeal membrane oxygenation (ECMO) in their unit. Epinephrine and norepinephrine were started in cold and warm shock, respectively. Further need for fluid boluses, vasoactive agents, and blood products was decided according to the type of septic shock, hemodynamic status, and fluid balance by the treating team. Data regarding demographics (age, gender, diagnosis, pediatric risk of mortality-PRISM III score) and clinical variables including duration of vasoactive therapy, the worst lactate value, need for blood transfusion, steroid, the central line used, and side effects of vasoactive therapy, ventilation and PICU outcome (survived/nonsurvived) were collected. IS and VIS were calculated using following formula, [IS $=$ Dobutamine dose $(\mu \mathrm{g} / \mathrm{kg} / \mathrm{min})+$ Dopamine dose $(\mu \mathrm{g} / \mathrm{kg} / \mathrm{min})+100 \times$ Epinephrine dose $(\mu \mathrm{g} / \mathrm{kg} / \mathrm{min})$ ] and [VIS $=\mathrm{IS}+10 \times$ Milrinone dose $(\mu \mathrm{g} / \mathrm{kg} / \mathrm{min})+10,000 \times$ Vasopressin dose (units $/ \mathrm{kg} /$ $\mathrm{min})+100 \times$ Norepinephrine dose $(\mu \mathrm{g} / \mathrm{kg} / \mathrm{min})]$ respectively $[5,7]$. The IS and VIS were calculated every $2 \mathrm{~h}$ or more frequently as indicated by dose change or addition of drugs from the documents. The mean IS, and VIS values were calculated for analysis purposes.

The distribution of data was assessed using the Kolmogorov-Smirnov $z$ test. The data were compared between survivors and nonsurvivors. Continuous data were compared using Student $t$-test if normally distributed or Mann-Whitney $U$ test if non-normally distributed. Qualitative data were compared using the Chi-square test (or Fisher exact test if cell frequencies were $<5)$. Receiver operating characteristic (ROC) curves and area under the curve (AUC) with $95 \%$ confidence interval were developed using mean IS and mean VIS value to predict the PICU mortality, and Youden index cut values were generated. Sensitivity and specificity (with a 95\% confidence interval) were calculated for the cutoff point of IS and VIS. Univariable followed by multivariable binary regression (Enter: Method) analysis using a Youden index cut points of IS and VIS determined from ROC were performed for PICU mortality, adjusting for age, gender, and illness severity (PRISM-III score). The final model fit was assessed using the Hosmer and Lemeshow test. The odds ratio with a $95 \%$ confidence interval was calculated. All tests were two-sided, and $p<0.05$ was considered statistically significant. IBM SPSS version 20.0 and MedCalc 19.6 versions were used for data analysis.

\section{Results}

A total of 176 patients were enrolled (survivor $n=72,41 \%$ and nonsurvivor $n=104,59 \%$ ) after the screening of 236 patients with septic shock (excluded: received $>6 \mathrm{~h}$ of vasoactive therapy, $n=33, \geq 2$ organ dysfunction, $n=27$ ). Demographic and clinical variables of enrolled patients were given in Table 1. All patients in the nonsurvivor group and 54 patients in the survivor group received mechanical ventilation. In the survivor group, 18 patients received non-invasive ventilation. ROC curve analysis to predict PICU mortality using mean IS had an area under the curve (AUROC) of 0.80 (95\%CI, 0.74-0.86; $p=<0.001$ ) [Youden index $J=0.4818$; sensitivity of 88.5 (95\% CI, 80.7-94) and specificity of 58.3 (95\% CI, 46.1-69.8)] (Fig. 1a). ROC curve analysis to predict PICU mortality using mean VIS had an AUROC of 0.88 (95\%CI, $0.82-0.92 ; p=<0.001$ ) [Youden index $J=0.6421$; sensitivity of 83.7 (95\% CI, 75.1-90.2) and specificity of 80.6 (95\% CI, 69.5-89) (Fig. 1b). The respective cutoff scores of IS and VIS were 28 and 42.5. On binary regression analysis, illness severity, worst lactate value, IS ( $>28)$, and VIS ( $>42.5)$ independently predicted the PICU mortality (Table 2). One patient had ventricular arrhythmia, and another patient had peripheral ischemic changes during the vasoactive agent administration.

\section{Discussion}

In this study, IS $>28$ and VIS $>42.5$ had good sensitivity and specificity for predicting mortality in pediatric septic shock with the area under the curve of 0.80 and 0.88 , respectively. In addition to IS and VIS, illness severity and worst lactate value independently predicted mortality. 
Table 1 Demographic and clinical variables of enrolled patients

\begin{tabular}{|c|c|c|c|}
\hline Variables & Survivor $(n=72)$ & Nonsurvivor $(n=104)$ & $p$ value \\
\hline${ }^{\#}$ Age, mo & $11(5-36)$ & $12(4-36)$ & $0.88^{\mathrm{a}}$ \\
\hline Male:Female, $n(\%)$ & $39(54.2): 33(45.8)$ & $59(56.7): 45(43.3)$ & $0.74^{\mathrm{b}}$ \\
\hline Age $<5$ y, $n(\%)$ & $60(83.3)$ & $87(83.6)$ & $0.96^{\mathrm{b}}$ \\
\hline${ }^{\#}$ Pediatric risk of mortality-III score & $14(10-18)$ & $22(18-28)$ & $<0.001^{\mathrm{a}}$ \\
\hline Co-morbidity, $n(\%)$ & $20(27.8)$ & $35(33.7)$ & $0.41^{\mathrm{b}}$ \\
\hline${ }^{\# \#}$ Lactate, $\mathrm{mmol} / \mathrm{L}$ & $3.2(1.9)$ & $6.1(3.6)$ & $<0.001^{\mathrm{c}}$ \\
\hline Focus/Diagnosis, $n(\%)$ & & & $0.13^{\mathrm{b}}$ \\
\hline Lung & $37(51.4)$ & $40(38.5)$ & \\
\hline Gastrointestinal & $9(12.5)$ & $9(8.6)$ & \\
\hline Central nervous system & $6(8.3)$ & $5(4.8)$ & \\
\hline Renal & $5(7)$ & $9(8.6)$ & \\
\hline Postoperative & $3(4.2)$ & $3(2.9)$ & \\
\hline Hematological & $2(2.8)$ & $11(10.6)$ & \\
\hline Cardiovascular & - & $4(3.8)$ & \\
\hline Others & $10(13.8)$ & $23(22.1)$ & \\
\hline Central line, $n(\%)$ & & & $<0.001^{\mathrm{b}}$ \\
\hline Internal jugular vein & $34(47.2)$ & $80(77)$ & \\
\hline Femoral vein & $19(26.4)$ & $21(20.2)$ & \\
\hline Internal jugular \& femoral vein* & $18(25)$ & $2(1.9)$ & \\
\hline Peripherally inserted central line & $1(1.4)$ & $1(0.9)$ & \\
\hline${ }^{\# \#}$ Fluid bolus, $\mathrm{mL} / \mathrm{kg}$ & $44.3(13)$ & $49(15.4)$ & $0.040^{\mathrm{c}}$ \\
\hline Blood transfusion, $n(\%)$ & $41(57)$ & $75(72.1)$ & $0.037^{\mathrm{b}}$ \\
\hline Steroid, $n(\%)$ & $22(30.6)$ & $70(67.3)$ & $<0.001^{\mathrm{b}}$ \\
\hline Kidney replacement therapy, $n(\%)$ & $12(16.7)$ & $23(22.1)$ & $0.37^{\mathrm{b}}$ \\
\hline${ }^{\# \#}$ Inotrope score & $23.2(14.6)$ & $42.2(15.4)$ & $<0.001^{\mathrm{c}}$ \\
\hline${ }^{\# \#}$ Vasoactive inotrope score & $31.4(24)$ & $75.2(29.6)$ & $<0.001^{\mathrm{c}}$ \\
\hline${ }^{\#}$ Duration of vasoactive-inotropic score, $\mathrm{h}$ & $25(12-69)$ & $34(18-70)$ & $0.24^{\mathrm{a}}$ \\
\hline${ }^{\#}$ Pediatric intensive care unit stay, $\mathrm{d}$ & $10(5-18)$ & $3(1-10)$ & $<0.001^{\mathrm{a}}$ \\
\hline${ }^{\#}$ Mechanical ventilation, day & $7(5-12)$ & $2(1-7)$ & $<0.001^{\mathrm{a}}$ \\
\hline
\end{tabular}

All data were presented number with percentage except \#median (IQR) or \#\#mean (SD)

*Patients underwent both central venous line at some point of time during PICU stay

${ }^{a}$ Mann-Whitney $U$ test

${ }^{\mathrm{b}} \mathrm{Chi}$-square test

${ }^{\mathrm{c}}$ Student $t$-test
There are limited studies that reported VIS and mortality in pediatric sepsis and septic shock $[12,16]$. Haque et al. [12], in a retrospective study involving 71 pediatric septic shock children, reported $100 \%$ mortality in those who had VIS $>20$ during the first $48 \mathrm{~h}$. Similar to the present study, this study was from an LMIC, and the baseline mortality was high (42\%). Nevertheless, in the present study, patients were sicker, and the authors took the mean IS and VIS values during the entire infusion duration rather than restricting them to the first $48 \mathrm{~h}$.

Similarly, McIntosh et al. [16] found a strong correlation between VIS at $48 \mathrm{~h}$ and PICU length of stay $(r=0.53)$ and ventilator days $(r=0.52)$. In contrast to the present study, the VIS at $48 \mathrm{~h}$ was not significantly associated with mortality and other composite outcomes. The study setting was different, as it was from a high-income country with a low (5.8\%) baseline mortality rate and access to ECMO service, so probably the analysis was underpowered to detect a significant difference.

In the present study, both IS and VIS predicted mortality independent of the validated PRISM-III score. The commonly used PRISM-III and PIM-2 scores were developed in diverse PICU patients and included the most abnormal parameters in the first $24 \mathrm{~h}$ of admission [17, 18]. These predictive scores also lack specificity to any organ dysfunction. The progression of cardiovascular dysfunction beyond $24 \mathrm{~h}$ might be revealed by continuous monitoring of IS and VIS, which is not fully addressed by the PRISM-III and PIM-2 score. Similarly, McIntosh et al. [16] showed the association of VIS at $48 \mathrm{~h}$ and short-term outcomes independent of PIM-3 score in pediatric sepsis.

The timing of IS and VIS calculation is an ongoing debate. In the postoperative cardiac setting, studies took the first 24 to $48 \mathrm{~h}$ as a reasonable period, and persisting 

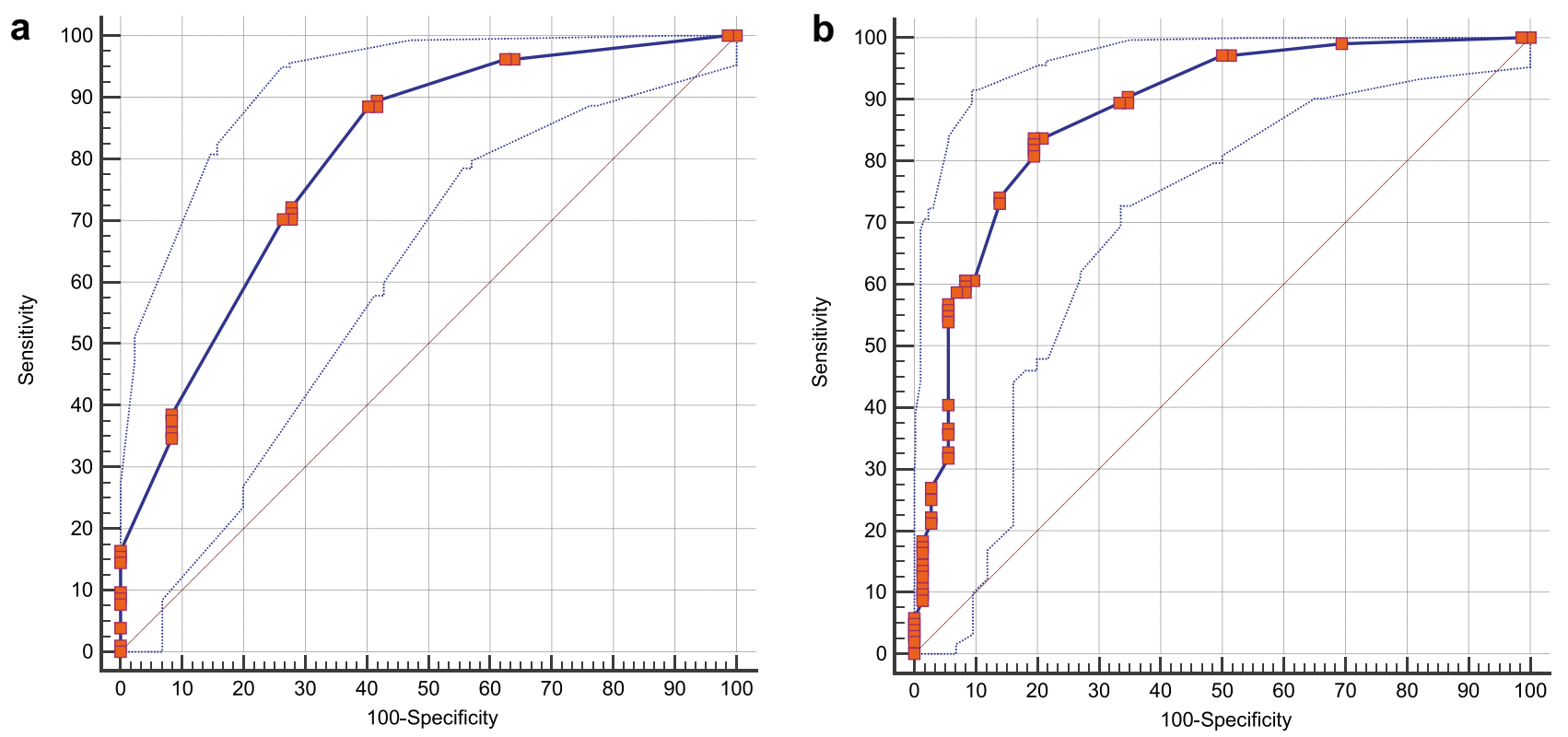

Fig. 1 a The area under receiver operating characteristic curve for inotropic score (IS) using cutpoint of 28 was 0.80 , and b for vasoactiveinotropic score (VIS) using cutpoint of 42.5 was 0.88

cardiovascular dysfunction beyond this period indicates poor outcomes [7, 19]. Few studies used VIS at $48 \mathrm{~h}$ to avoid the effect of aggressive resuscitation and rapid escalation of vasoactive in the initial hours for predicting the outcomes in pediatric sepsis [16]. The initial VIS during the early hours of resuscitation may be falsely high. The clinical experience also supports this concept that de-escalation of vasoactive therapy is often possible at the end of $48 \mathrm{~h}$ if early resuscitation is initiated.

Musick et al. [9], in a retrospective study involving 2752 PICU patients, derived ROC curves at various time points (maximum score in $24 \mathrm{~h}, 48 \mathrm{~h}$, any period, VIS at $24 \mathrm{~h}$, VIS at $48 \mathrm{~h}$ ) for predicting the mortality. AUC was almost similar for the maximum score during the initial course and VIS at $48 \mathrm{~h}(0.788$ and 0.736$)$. However, the study population included heterogeneous PICU patients, and VIS was calculated after documented dose change of vasoactive medications. In the present study, the authors calculated IS and VIS during the entire duration of vasoactive infusion and took the mean value as a surrogate score of overall cardiovascular support during the entire PICU stay. The AUC for predicting the mortality by IS and VIS score in the present study are 0.80 and 0.88 , respectively, which are higher than the other studies.

On multivariable analysis, only severity (PRISM-III score), lactate, IS $>28$, VIS $>42.5$ were independently predicted the
Table 2 Multivariable binary regression analysis for predictors of mortality in children with septic shock

\begin{tabular}{lclclll}
\hline Variables & $\begin{array}{l}\text { Unadjusted } \\
\text { odds ratio }\end{array}$ & $\begin{array}{l}\text { 95\% confi- } \\
\text { dence interval }\end{array}$ & $p$ value & $\begin{array}{l}\text { Adjusted } \\
\text { odds ratio }\end{array}$ & $\begin{array}{l}\text { 95\% confi- } \\
\text { dence interval }\end{array}$ & $p$ value \\
\hline Age & 1.00 & $0.99-1.01$ & 0.92 & 1.00 & $0.99-1.01$ & 0.66 \\
PRISM-III & 1.15 & $1.09-1.21$ & $<0.001$ & 1.12 & $1.05-1.12$ & 0.001 \\
Lactate & 1.58 & $1.32-1.88$ & $<0.001$ & 1.31 & $1.08-1.58$ & 0.005 \\
Blood transfusion & 1.96 & $1.04-3.68$ & 0.037 & 1.09 & $0.39-3.01$ & 0.87 \\
Steroid received & 4.68 & $2.45-8.94$ & $<0.001$ & 1.81 & $0.67-4.89$ & 0.25 \\
Fluid bolus received & 1.02 & $1.00-1.05$ & 0.042 & 1.00 & $0.97-1.04$ & 0.95 \\
Inotropic score $>28$ & 11.37 & $5.30-24.41$ & $<0.001$ & 3.98 & $1.24-12.80$ & 0.021 \\
Vasoactive-inotropic & 21.20 & $9.70-46.32$ & $<0.001$ & 4.66 & $1.57-13.87$ & 0.006 \\
$\quad$ & & & & & & \\
\hline
\end{tabular}

Variables entered in model are age (continuous variable), PRISM-III (continuous variable), lactate (continuous variable), blood transfusion (Yes/No), steroid received (Yes/No), fluid bolus received (continuous variable), inotropic score (cutoff point 28), and Vasoactive-inotropic score (cut-off point 42.5). Hosmer and Lemeshow goodness of fit model $p=0.319$. Overall percentage of model is $84 \%$ with $r^{2}$ value of 0.625

PRISM Pediatric Risk of Mortality 
mortality. The mean fluid bolus received was 44.3 vs. 49 (mL/ $\mathrm{kg}$ ) among survivors and nonsurvivors, respectively, which is lower than the recommendation by ACCM guidelines [14]. Although the amount of fluid bolus received was significantly higher among the nonsurvivors, it did not independently predict the mortality after adjusting the confounders.

The present study's unique aspect is that the study population had high sepsis-related baseline mortality, similar to the other LMIC, and enrolled a reasonable sample size. The authors calculated IS and VIS during the entire duration of vasoactive infusion rather than restricting it to a particular time. The mean IS and VIS provides the overall picture of cardiovascular support during the PICU stay. The present study population was restricted to sepsis rather than the general PICU population, where many well-validated prognostic scores are available $[17,18]$. There are a few limitations. First, it is a single-center, retrospective study. Fluid bolus and vasoactive medication usage may vary according to institutional and personal preferences. So the results may not be generalizable to other populations. Second, the calculation of IS and VIS includes commonly used inotropes and vasoactive medications. Nevertheless, other agents like levosimendan, phenylephrine were not taken into account in the calculation. However, these agents are not widely used in septic shock. Third, the authors did not compare the performance of these scores between patients with or without myocardial dysfunction. Fourth, long-term functional outcomes are not studied. The prospective, multicentric studies focusing on the discrete patient subgroups (myocardial dysfunction in sepsis), correlation with ECHO, and long-term follow-up are needed in the future to address these limitations.

\section{Conclusion}

The study concludes that the threshold of IS ( $>28$ ) and VIS $(>42.5)$ independently predicted the mortality in pediatric septic shock. Besides IS and VIS, PRISM-III and the worst lactate value also independently predicted pediatric septic shock mortality in PICU.

Acknowledgements The authors acknowledge the contribution of Mrs. S. Raja Deepa B.Com, MCA (JIPMER Campus, Puducherry, India) for data handling, review and editing of the manuscript; Mr. Rakesh Mohindra (Punjab University, Chandigarh, India) and Mrs. Thenmozhi M (M.Sc, Ph.D., Senior demonstrator, Department of Biostatistics, $\mathrm{CMC}$, Vellore, India) helping with statistical analysis and Mrs. Harpreet Kaur (Punjab University, Chandigarh, India), and Mrs. Neelima Chadha (Tulsi Das Library, PGIMER, Chandigarh, India) helping with medical literature search.

Authors' Contribution RR: Conceptualized the study, reviewed the literature and critically reviewed the manuscript; DK, MK: Data collection, reviewed the literature and contribution of manuscript writing;
SM: Protocol development, review of literature and manuscript writing; KK, TS: Data analysis and interpretation, review of literature and manuscript writing; RR, SM: Involved in the management of the patients. All authors approved the final version of the manuscript. Study supervision: RR, SM. RR is the guarantor of the paper.

Funding Supported in part by the institutional and departmental fund.

\section{Declarations}

Ethics Committee Approval Obtained (Approval No. JIP/IEC/2017/ 0464).

Conflict of Interest None.

\section{References}

1. Liu L, Chu Y, Oza S, et al. National, regional, and state-level allcause and cause-specific under-5 mortality in India in 2000-15: a systematic analysis with implications for the sustainable development goals. Lancet Glob Health. 2019;7:e721-34.

2. Kaur G, Vinayak N, Mittal K, Kaushik JS, Aamir M. Clinical outcome and predictors of mortality in children with sepsis, severe sepsis, and septic shock from Rohtak, Haryana: A prospective observational study. Indian J Crit Care Med. 2014;18:437-41.

3. Maitland K, Kiguli S, Opoka RO, et al. Mortality after fluid bolus in African children with severe infection. N Engl J Med. 2011;364:2483-95.

4. Jain A, Sankar J, Anubhuti A, Yadav DK, Sankar MJ. Prevalence and outcome of sepsis-induced myocardial dysfunction in children with "sepsis" "with" and 'without shock'-a prospective observational study. J Trop Pediatr. 2018;64:501-9.

5. Wernovsky G, Wypij D, Jonas RA, et al. Postoperative course and hemodynamic profile after the arterial switch operation in neonates and infants. a comparison of low-flow cardiopulmonary bypass and circulatory arrest. Circulation. 1995;92:2226-35.

6. Williams FZ, Sachdeva R, Travers CD, Walson KH, Hebbar KB. Characterization of myocardial dysfunction in fluid- and catecholaminerefractory pediatric septic shock and its clinical significance. J Intensive Care Med. 2019;34:17-25.

7. Gaies MG, Gurney JG, Yen AH, et al. Vasoactive-inotropic score as a predictor of morbidity and mortality in infants after cardiopulmonary bypass. Pediatr Crit Care Med. 2010;11:234-8.

8. Koponen T, Karttunen J, Musialowicz T, Pietilainen L, Uusaro A, Lahtinen P. Vasoactive-inotropic score and the prediction of morbidity and mortality after cardiac surgery. Br J Anaesth. 2019;122:428-36.

9. Musick MA, Loftis LL, Kennedy CE. Comparing vasoactive-inotropic score reporting strategies in the PICU relative to mortality risk. Pediatr Crit Care Med. 2018;19:1130-6.

10. Gulla KM, Sachdev A, Gupta D, Gupta N, Anand K, Pruthi PK. Continuous renal replacement therapy in children with severe sepsis and multiorgan dysfunction - a pilot study on timing of initiation. Indian J Crit Care Med. 2015;19:613-7.

11. Kawai Y, Cornell TT, Cooley EG, et al. Therapeutic plasma exchange may improve hemodynamics and organ failure among children with sepsis-induced multiple organ dysfunction syndrome receiving extracorporeal life support. Pediatr Crit Care Med. 2015;16:366-74.

12. Haque A, Siddiqui NR, Munir O, Saleem S, Mian A. Association between vasoactive-inotropic score and mortality in pediatric septic shock. Indian Pediatr. 2015;52:311-3. 
13. Rhodes A, Evans LE, Alhazzani W, Levy MM, Antonelli M, Ferrer $\mathrm{R}$, et al. Surviving Sepsis Campaign: International Guidelines for Management of Sepsis and Septic Shock: 2016. Intensive Care Med. 2017;43:304-77.

14. Davis AL, Carcillo JA, Aneja RK, et al. American college of critical care medicine clinical practice parameters for hemodynamic support of pediatric and neonatal septic shock. Crit Care Med. 2017;45:1061-93.

15. Goldstein B, Giroir B, Randolph A. International Consensus Conference on Pediatric S. International pediatric sepsis consensus conference: definitions for sepsis and organ dysfunction in pediatrics. Pediatr Crit Care Med. 2005;6:2-8.

16. McIntosh AM, Tong S, Deakyne SJ, Davidson JA, Scott HF. Validation of the vasoactive-inotropic score in pediatric sepsis. Pediatr Crit Care Med. 2017;18:750-7.
17. Pollack MM, Patel KM, Ruttimann UE. PRISM III: an updated pediatric risk of mortality score. Crit Care Med. 1996;24:743-52.

18. Slater A, Shann F, Pearson G. Paediatric Index of Mortality Study G. PIM2: a revised version of the paediatric index of mortality. Intensive Care Med. 2003;29:278-85.

19. Gaies MG, Jeffries HE, Niebler RA, et al. Vasoactive-inotropic score is associated with outcome after infant cardiac surgery: an analysis from the pediatric cardiac critical care consortium and virtual PICU system registries. Pediatr Crit Care Med. 2014;15:529-37.

Publisher's Note Springer Nature remains neutral with regard to jurisdictional claims in published maps and institutional affiliations. 